\title{
THE BEGINNING AND THE END OF THE INSECTS' FLIGHT TOWARDS THE LIGHT ACCORDING TO DIFFERENT ENVIRONMENTAL LIGHTINGS
}

\author{
L. NOWINSZKY ${ }^{* 1}-$ Z. MÉSZÁROS ${ }^{2}-$ J. PUSKÁS ${ }^{1}$ \\ ${ }^{1}$ Berzsenyi Dániel College, 9701 Szombathely, Károlyi Gáspár Square 4. \\ (phone: +36-94-504-357) \\ ${ }_{2}^{2}$ Plant Protection Research Institute of Hungarian Academy of Sciences, \\ 1025 Budapest, Herman Ottó Str.15. \\ *Corresponding author \\ e-mail:nlaszlo@bdf.hu \\ (Received $12^{\text {nd }}$ June 2008 ; accepted $23^{\text {rd }}$ September 2008)
}

\begin{abstract}
Many news bulletins found in the literature only consent themselves with the description of the night distribution of the trapped species not examining the beginning and the end of the insects' flight towards the light with the measurement of environmental lighting, expressed in lux. For this reason, we had examined the daily appearance of the first and last specimens of the species in the light trap concerning the exact lighting figures. We have used the hourly collection data of the fractionating light trap at the farm of Julianna in Nagykovácsi, belonging to the MTA Crop Protection Research Institute. With the help of our own computer programme we had counted the light coming from the sun, moon and from the starry sky, for every full hour separately and in total. We had given two lighting figures to every trapping data: we had counted the first minute of the first and the next hour of the given hour in lux within which the trapping had happened. Hereby, two lighting figures had become known during which the flight towards lighting had begun and end. The flight of 51 species towards the light happens when the total of the given hour concur in the duration of navigation twilight, of 26 species in the duration of sidereal twilight and of 7 species in the duration of night light. There were 2 species where the first imago was already captured during daylight. In the period of the quick reduction of lighting only the first specimens of the 14 species appear, accordingly: 4 from daylight to civil twilight, 4 from daylight to navigation twilight, 1 from sunset to navigation twilight and 4 species from civil twilight to sidereal twilight. The flight towards light ends in the case of 16 species after midnight during the night light, at 48 species during sidereal twilight and at 28 species at navigation twilight. The flight of only 3 species end when it is clearing up quicker within the given hour, from navigation twilight to daylight. Our results could stop a gap.
\end{abstract}

Keywords: Lepidoptera, flying threshold, lux

\section{Introduction and survey of literature}

The majority of researchers do not examine the beginning and the end of the insects' flight towards the light with the measurement of environmental lighting, expressed in lux. Most of the authors were satisfied to describe the night distribution of the trapped insects. We only sporadically find records in the literature in reference to at how much lux lighting we can experience the start of certain insects' flight.

According to Mazorchin-Porsnjakov [1] the species of the chestnut cockchafer (Melolontha hippocastani Fabr.) start flying at 14 lux but can only be collected with light trap when lighting is decreased to 7-8 lux.

Tshernyshev [2] had carried out light-trap collectings and visual observations around Moscow. In reference to many insect classes and many important species, he also 
reported the associated measurement of lighting in lux to the start and maximum of activity.

- Ephemeroptera: Ordella horaria L. 4-0.005 lux;

- Homoptera: Psylla betulae L. 0.001 lux;

- Heteroptera: Sigara falleni Fieb.. S. striata L.. S. praeusta Fieb. 10 lux;

- Coleoptera: Amara majuscula Chd. 1-0.5 lux, Hybius ater DeGeer 0.01 lux, Hydrobius fuscipes L. 1 lux, Cercyon quisquillus L., C. haemorrhoidalis Fabr., C. unipunctatus L., C. melanocephalus L. 5-1 lux, Serica brunnea L. 0.001 lux, Necrophorus vespillo L. 10-0.1 lux, Oxytellus rugosus L. 1 lux, Bledius opacus Block. and Heterocerus hispidulus Kieff. 100-10 lux, Aphodius rufus Müll. A. distinctus Müll. 10-1 lux, but these last mentioned species had flew at 10.000 lux as well in May, daytime at 15-17 degrees Celsius.

- Hymenoptera: Ophion luteus L. 0.01 lux, Lasius niger L. 1000-50 lux;

- Diptera: Culicoides pulicaris L. and C. grisescens Edw. 10-0.03 lux;

- Trichoptera: Psychomya pusilla Fbr., Leptocerus dissimilis Steph., L. nigriversosus Retz., Hydropsyche ornatula McLeach., Halesus interpunctatus Zett. 0.1-0.01 lux;

- Lepidoptera: most of the species between 0.01-0.001 lux, but Hepialus sylvinus L., H. humuli L. 5-1 lux.

Skuhravý and Zumr [3] had studied the night activities of the black-arches moth (Lymantria monacha L.) in pine tree stand in the Czech Republic. Their flight towards light had began when lighting decreased to 1-3 lux. Through Dreisig's [4] studies in Denmark and Florida he had allocated that the beginning of activities of certain species are specific, usually starts between 1 and 0,003 lux at invariant lighting. If the period of twilight increases, the dispersion of the invariant rate will be higher. This is also influenced by the season besides the geographical latitude. He also gives data about the beginning of the flight of the Macrolepidoptera species related to the environmental lighting. These are the following in Denmark: Plusia gamma L. 1-12 lux, Agrotis exclamationis L. 0.4 lux, Caradrina morpheus Hfn. 0.15 lux, Typhena pronuba L. 0.9 lux, Monima pulverulenta Esp. 0.09 lux, Cerapteryx graminis L. 0.8 lux, Deilephia porcellus L. 1.4 lux, Malacosoma castrensis L. 0.5 lux. In Florida: Plusia gamma L. 0.8 lux, Heliothis virescens Fabr. 0.03 lux, Spodoptera frugiperda Smith 0.007 lux, Anticarsia gemmalis Hbn. 0.08 lux, Mocis latipes Guenee 0.009 lux, Schinia nubila Str. 0.15 lux, Megalopyge opercularis Abbot \& Smith 0.02 lux, Nystalea sp. 0.004 lux.

As there are not too many data in literature about the beginning and the end of flight towards the environmental light that is given is lux, we have examined the day-by-day appearance of the first and last specimens of certain species using our domestically collected figures.

\section{Materials}

At the Crop Protection Research Institute near Budapest, we had operated a fractionating light trap between 1976-1979 at the research plant of Julianna, in Nagykovácsi and the insects collected there had been separated into different flasks. The light trap had operated with a 125-watt HGL bulb. However, this light trap did not operate every night, only periodically, 57 times altogether but that time for 12 hours, in the spring and summer time, early in the autumn from 5 p.m. to 5 a.m. and in the second 
half of October from 4 p.m. to 4 a.m. (UT). These years the daylight saving time was not applied. Mészáros had defined and journalized from the collected insects the insect pests from all Macrolepidoptera and Microlepidoptera species. We had used the records of 160 Lepidoptera- and 1 Coleoptera species for our work.

The operation had taken part on the following days:

1976: 08. 26-27, 10. 06-07;

1977. 03.10-11, 03.14-15, 03.16-17, 03.17-18, 03.18-19, 03.19-20, 03.21-22, 03.22-23, 03.24-25, 03.28-29, 04.14-15, 04.18-19, 04.19-20, 04.20-21, 04.22-23, 04.26-27, 04.28-29, 05.04-05, 05.09-10, 05.12-13, 05.16-17, 05.19-20, 05.28-29, 05.30-31, 06.02-03, 06.06-07, 06.09-10, 06.13-14, 06.16-17, 06.21-22, 06.24-25, 06.28-29, 07.04-05, 07.12-13, 07.26-27, 07.27-28, 08.08-09, 09.06-07, 09.15-16, 09.22-23, $10.06-07,10.13-14,10.20-21,10.2-28$

1978. 09.18-19, 09.26-27, 11.02-03

1979. 03.22-23, 03.28-29, 05.10-11, 05.17-18, 05.25-26, 06.20-21, 06.22-23, 07.23-24.

We had counted the lighting data required for our examinations with the help of our own computer programme. György Tóth, astronomer - who unfortunately cannot be with us any longer - for a TI 59, established this programme. Computer, to be used in our common researches (Nowinszky and Tóth, [5]). This programme was adapted to a modern computer by Miklós Kiss, associate professor, for which hereby we would like to express our thanks.

The programme counts the daytime and twilight lighting from the sun to optional geographical place, day and time separately and in total, the light of the moon if it is over the horizon and the lighting coming from the starry sky, all these data in lux. It also takes the number of clouds into consideration when counting.

We had collected every data concerning all clouds from the yearbooks of the National Meteorological Organization. In these, data is recorded every 3 hours with causal explanation. We had applied the data to the related given hour and to the next 2 hours.

\section{Methods}

We have collected the hour of the capture of the first and last specimens of the trapped species from the light trap journal in reference to every night. We have counted the full lighting figures of these time periods. As, of course, the exact trapping time is unknown the lighting figures were counted in reference to a whole hour. We had given two lighting figures to every trapping data: we had counted the first minute of the first and the next hour of the given hour in lux, within which the trapping had happened. Hereby, two lighting figures had become known during which the flight towards lighting had begun and end.

By species, we had put the lux value pairs into order according to the first and last figures of trapping. Our figures were put into a table. We had placed those lighting value pairs into this table, in which the first specimen is already, the last still flew, also separated accordingly whether the trapping had happened before or after midnight. 


\section{Results}

Table 1. contains the figures of 161 species. We had also aspired to include as many information as possible. To achieve this we had also given to every lux value the period of twilight or night, it belonged to. The abbreviations of these are the following: the numbers in italics show the trappings after midnight, $*=$ only one figure apply to a given specimen, $\mathrm{T} / \mathrm{N}=$ twilight or night hour, $\mathrm{D}=$ daylight, $\mathrm{S}=$ sunset, $\mathrm{C}=$ civil twilight, $\mathrm{A}=$ sidereal twilight, $\mathrm{NS}=$ the light of the night sky

\section{Discussion}

The beginning of flight towards the light at night happens at distinct lighting conditions in the case of certain species. These do not indicate lawfulness that should be linked to taxonomical rating. The flight of 50 species towards the light happens when the total of the given hour concur in the duration of navigation twilight, of 26 species in the duration of sidereal twilight and of 7 species in the duration of night light. There were 2 species where the first imago was already captured during daylight. In the period of the quick reduction of lighting only the first specimens of the 14 species appear, accordingly: 4 from daylight to civil twilight, 4 from daylight to navigation twilight, 1 from sunset to navigation twilight and 4 species from civil twilight to sidereal twilight.

The flight towards light ends in the case of 16 species after midnight during the night light, at 48 species during sidereal twilight and at 28 species at navigation twilight. The flight of only 3 species end when it is clearing up quicker within the given hour, from navigation twilight to daylight.

Although we only have a few results, many of these are often from one collection figure, we believe it is worth to share with our readers. On the one hand because we could not find any researches like this in the literature, which publish the flight peculiarities of so many species, on the other hand because those are also not from mass collection figures. Of course, our published results will be altered by our continuous observations but until then with their informative nature can stop a gap. We could get interesting informations for example from entomologists who should journalize the exact arrival time of the insects into the capturing sheet and should also measure the lighting related to it. The measuring instrument needed for this is fairly easy to access nowadays and although they are not occupied with, researches like this could help the entomological studies with very important and precise data. 
Table 1. Beginning and ending of flight of Lepidoptera species before and after midnight in connection with the twilights

\begin{tabular}{|c|c|c|c|c|c|c|c|c|}
\hline \multirow{2}{*}{$\begin{array}{c}\text { Species } \\
\text { Lepidoptera }\end{array}$} & \multicolumn{4}{|c|}{$\begin{array}{c}\text { Beginning and ending of flight } \\
\text { (before midnight) } \\
\text { between }\end{array}$} & \multicolumn{4}{|c|}{$\begin{array}{c}\text { Ending of flight (before or } \\
\text { after midnight) } \\
\text { between }\end{array}$} \\
\hline & Lux & $T / N$ & Lux & $T / N$ & Lux & $T / N$ & Lux & $T / N$ \\
\hline \multirow{6}{*}{$\begin{array}{l}\text { Plutellidae } \\
\text { Plutella maculipennis Curt. } \\
\text { Gelechiidae } \\
\text { Anarsia lineatella Zeller* } \\
\text { Recurvaria leucatella Clerck. } \\
\text { Recurvaria nanella Hbn. } \\
\text { Sitochroga verticalis L. }\end{array}$} & & & & & & & & \\
\hline & 79.22 & $\mathrm{C}$ & 0.1450 & $\mathrm{~N}$ & 0.0019 & A & 0.0071 & $\mathrm{~A}$ \\
\hline & 0.0384 & $\mathrm{~N}$ & 0.0388 & $\mathrm{~N}$ & & & & \\
\hline & 0.0028 & A & 0.0031 & A & 0.0987 & $N$ & 1.821 & $C$ \\
\hline & 0.0388 & $\mathrm{~N}$ & 0.0391 & $\mathrm{~N}$ & 0.0039 & $\mathrm{~A}$ & 0.0029 & A \\
\hline & 0.1450 & $\mathrm{~N}$ & 0.1003 & $\mathrm{~N}$ & 0.0203 & $N$ & 1.999 & $N$ \\
\hline \multicolumn{9}{|l|}{ Tortricidae } \\
\hline Pandemis heparana Schiff. & 1386.2 & $\mathrm{D}$ & 30.338 & $\mathrm{C}$ & 0.0017 & A & 0.0017 & A \\
\hline Pandemis ribeana $\mathrm{Hbn}$ & 1386.20 & $\mathrm{D}$ & 41.614 & $\mathrm{C}$ & 0.0717 & $\mathrm{~N}$ & 0.0714 & $\mathrm{~N}$ \\
\hline Argyrotaenia pulchellana Haw. * & & & & & 0.0396 & $N$ & 0.0472 & $N$ \\
\hline Adoxophyes reticulana $\mathrm{Hbn}$. & 0.0388 & $\mathrm{~N}$ & 0.0391 & $\mathrm{~N}$ & 0.0039 & $A$ & 1.001 & $N$ \\
\hline Hedya nubiferana Haw. & 649.680 & $\mathrm{D}$ & 2.5273 & $\mathrm{~N}$ & 0.0422 & $N$ & 90.57 & $C$ \\
\hline Spilonota ocellana F. & 12.6161 & $\mathrm{C}$ & 0.0114 & $\mathrm{~N}$ & 0.0019 & $A$ & 0.0071 & $A$ \\
\hline Cydia pomonella L. & 737.302 & $\mathrm{D}$ & 0.3745 & $\mathrm{~N}$ & 0.0276 & $N$ & 186.47 & $C$ \\
\hline Tortrix viridana L. $*$ & 0.0604 & $\mathrm{~N}$ & 0.0216 & $\mathrm{~N}$ & & & & \\
\hline \multicolumn{9}{|l|}{ Phycitidae } \\
\hline Oncocera semirubella Scop. & 0.0495 & $\mathrm{~N}$ & 0.0453 & $\mathrm{~N}$ & 0.0012 & $A$ & 0.0039 & $A$ \\
\hline Etiella zinckenella Tr. & 0.0195 & $\mathrm{~N}$ & 0.0178 & $\mathrm{~N}$ & 0.0022 & A & 0.0031 & A \\
\hline \multicolumn{9}{|l|}{ Pyraustidae } \\
\hline Ostrinia nubilalis Hbn. & 0.0021 & A & 0.0021 & A & 0.0045 & $A$ & 0.0067 & $A$ \\
\hline Loxostege sticticalis L. * & 0.0029 & $\mathrm{~A}$ & 0.0028 & A & & & & \\
\hline Evergestis extimalis Scop. & 0.0708 & $\mathrm{~N}$ & 0.0717 & $\mathrm{~N}$ & 0.0104 & $N$ & 0.0086 & $A$ \\
\hline Evergestis frumentalis L. & 0.0960 & $\mathrm{~N}$ & 0.0495 & $\mathrm{~N}$ & 0.0195 & $\mathrm{~N}$ & 0.0178 & $\mathrm{~N}$ \\
\hline \multicolumn{9}{|l|}{ Geometridae } \\
\hline Alsophila aescularia Schiff. & 346.480 & $\mathrm{C}$ & 0.0451 & $\mathrm{~N}$ & 0.0005 & NS & 0.0005 & NS \\
\hline Aplocera plagiata L. & 0.0101 & $\mathrm{~A}$ & 0.0094 & A & 0.0012 & $A$ & 0.0012 & $A$ \\
\hline Operophthera brumata L. & 0.0014 & A & 0.0014 & A & 0.0368 & $N$ & 0.0357 & $N$ \\
\hline Philereme vetulata Schiff. & 30.3375 & $\mathrm{C}$ & 0.0279 & $\mathrm{~N}$ & 0.0363 & $\mathrm{~N}$ & 0.0165 & $\mathrm{~N}$ \\
\hline Lygris pyraliata Schiff. & 0.0495 & $\mathrm{~N}$ & 0.0453 & $\mathrm{~N}$ & 0.0111 & $\mathrm{~N}$ & 0.0111 & $\mathrm{~N}$ \\
\hline Cidaria fulvata $\mathrm{L}$. & 0.0165 & $\mathrm{~N}$ & 0.0111 & $\mathrm{~N}$ & 0.0021 & A & 0.0021 & A \\
\hline Xanthorrhoe fluctuata L. * & 0.0008 & NS & 0.0004 & NS & & & & \\
\hline Hydrelia flammeolaria Hfn. * & 0.0025 & A & 0.0021 & A & & & & \\
\hline Eupithecia centaureata Schiff. * & 0.0717 & $\mathrm{~N}$ & 0.0714 & $\mathrm{~N}$ & & & & \\
\hline Bapta temerata Schiff. * & 0.0034 & A & 0.0008 & NS & & & & \\
\hline Ennomos erosaria Schiff. * & 0.1808 & $\mathrm{~N}$ & 0.1385 & $\mathrm{~N}$ & & & & \\
\hline Colotois pennaria L. * & & & & & 0.0014 & A & 0.0012 & $A$ \\
\hline
\end{tabular}




\begin{tabular}{|c|c|c|c|c|c|c|c|c|}
\hline \multirow{2}{*}{$\begin{array}{c}\text { Species } \\
\text { Lepidoptera }\end{array}$} & \multicolumn{4}{|c|}{$\begin{array}{c}\text { Beginning and ending of flight } \\
\text { (before midnight) } \\
\text { between }\end{array}$} & \multicolumn{4}{|c|}{$\begin{array}{c}\text { Ending of flight (before or } \\
\text { after midnight) } \\
\text { between }\end{array}$} \\
\hline & Lux & $T / N$ & Lux & $T / N$ & Lux & $T / N$ & Lux & $T / N$ \\
\hline Crocallis elinguaria L. * & 0.0008 & NS & 0.0008 & NS & & & & \\
\hline Plagodis dolabraria L. * & 0.0321 & $\mathrm{~N}$ & 0.0346 & $\mathrm{~N}$ & & & & \\
\hline Macaria alternaria Hbn. * & 0.0178 & $\mathrm{~N}$ & 0.0164 & $\mathrm{~N}$ & & & & \\
\hline Chiasmia clathrata L. & 0.8153 & $\mathrm{~N}$ & 0.0004 & NS & 0.8153 & $N$ & 611.76 & \\
\hline Erannis leucophaearia Schiff. * & 0.0211 & $\mathrm{~N}$ & 0.0026 & $\mathrm{~A}$ & & & & \\
\hline Erannis marginaria Bkh. & 0.0332 & $\mathrm{~N}$ & 0.0238 & $\mathrm{~N}$ & 0.0005 & NS & 0.0005 & NS \\
\hline Apocheima hispidaria Schiff. & 346.480 & $\mathrm{C}$ & 0.0451 & $\mathrm{~N}$ & 0.0006 & NS & 0.0006 & NS \\
\hline Nyssia zonaria Schiff. & 346.480 & $\mathrm{C}$ & 0.0451 & $\mathrm{~N}$ & 0.0010 & $A$ & 0.0010 & $A$ \\
\hline Lycia hirtaria $\mathrm{Cl}$. & 2853.20 & $\mathrm{D}$ & 17.124 & $\mathrm{C}$ & 0.0010 & $A$ & 0.0010 & $A$ \\
\hline Biston stratarius Hfn. & 346.480 & $\mathrm{C}$ & 0.0451 & $\mathrm{~N}$ & 0.0008 & NS & 0.0008 & NS \\
\hline Biston betularius L. & 0.0631 & $\mathrm{~N}$ & 0.0009 & NS & 0.0425 & $\mathrm{~N}$ & 110.41 & $\mathrm{C}$ \\
\hline Boarmia rhomboidaria Schiff. & 32.504 & $\mathrm{C}$ & 0.0311 & $\mathrm{~N}$ & 0.0031 & $A$ & 0.0048 & $A$ \\
\hline Boarmia cinctaria Schiff. $*$ & 0.1389 & $\mathrm{~N}$ & 0.1569 & $\mathrm{~N}$ & & & & \\
\hline Biston arenaria Hfn. & 0.0014 & A & 0.0006 & NS & 0.0007 & NS & 0.0007 & NS \\
\hline Ascotis selenaria Schiff. * & 0.0128 & $\mathrm{~N}$ & 0.0118 & $\mathrm{~N}$ & & & & \\
\hline Ectropis bistortata Goeze * & & & & & 0.0054 & $A$ & 0.0063 & $A$ \\
\hline Ematurga atomaria L. $*$ & & $\mathrm{~N}$ & 0.0040 & A & & & & \\
\hline Siona lineata L. & 0.0008 & NS & 0.0004 & NS & 0.0717 & $\mathrm{~N}$ & 0.0714 & $\mathrm{~N}$ \\
\hline Noctuidae & & & & & & & & \\
\hline Colocasia coryli L. * & & & & & 0.0840 & $N$ & 0.0888 & $N$ \\
\hline Apatele rumicis L. & 0.5266 & $\mathrm{~N}$ & 0.0086 & $\mathrm{~A}$ & 0.0006 & NS & 0.0003 & NS \\
\hline Euxoa temera $\mathrm{Hb} .{ }^{*}$ & 0.1258 & $\mathrm{~N}$ & 0.0228 & $\mathrm{~A}$ & & & & \\
\hline Euxoa obelisca Schiff. & 0.0029 & A & 0.0028 & $\mathrm{~A}$ & 0.0070 & $A$ & 0.0055 & $A$ \\
\hline Agrotis ypsilon Rott. & 0.0960 & $\mathrm{~N}$ & 0.0495 & $\mathrm{~N}$ & 0.0015 & A & 0.0015 & A \\
\hline Scotia segetum Schiff. & 32.5042 & $\mathrm{C}$ & 0.0311 & $\mathrm{~N}$ & 0.0086 & $A$ & 0.0088 & $A$ \\
\hline Scotia exclamationis L. & 2.5273 & $\mathrm{~N}$ & 0.0034 & $\mathrm{~A}$ & 0.0857 & $N$ & 0.5864 & $N$ \\
\hline Eugnorisma depuncta L. * & 0.0134 & $\mathrm{~N}$ & 0.0106 & $\mathrm{~N}$ & & & & \\
\hline Diarsia rubi View. * & 0.0604 & $\mathrm{~N}$ & 0.0216 & $\mathrm{~N}$ & & & & \\
\hline Xestia c-nigrum L. & 46.006 & $\mathrm{C}$ & 0.0604 & $\mathrm{~N}$ & 0.0007 & NS & 0.0007 & NS \\
\hline Epipsilia grisescens F. * & & & & & 0.0004 & $N S$ & 0.0008 & $N S$ \\
\hline Ochropleura plecta L. & 0.0063 & A & 0.0061 & $\mathrm{~A}$ & 0.0013 & A & 0.0007 & NS \\
\hline Diarsia rhomboidea Schiff. & 0.0028 & A & 0.0026 & $\mathrm{~A}$ & 0.0009 & NS & 0.0009 & NS \\
\hline Diarsia xanthographa Schiff. * & 0.0057 & A & 0.0045 & $\mathrm{~A}$ & & & & \\
\hline Cerastis rubricosa Schiff. & 17.1235 & $\mathrm{C}$ & 0.0049 & A & 0.0124 & $N$ & 1.6583 & $N$ \\
\hline Ammoconia caecimacula Schiff. & 18.092 & $\mathrm{C}$ & 0.1921 & $\mathrm{~N}$ & 0.0037 & $A$ & 0.0029 & $A$ \\
\hline Noctua pronuba L. & 16.6122 & $\mathrm{C}$ & 0.0134 & $\mathrm{~N}$ & 0.0009 & NS & 0.0012 & A \\
\hline Triphaena orbona Hfn. & 0.0057 & A & 0.0045 & A & 0.0012 & $A$ & 0.0012 & $A$ \\
\hline Mamestra brassicae Hfn. & 0.0142 & $\mathrm{~N}$ & 0.0128 & $\mathrm{~N}$ & 0.0007 & NS & 0.0007 & NS \\
\hline Mamestra suasa Schiff. & 0.0128 & $\mathrm{~N}$ & 0.0118 & $\mathrm{~N}$ & 0.0054 & $A$ & 0.0063 & $A$ \\
\hline
\end{tabular}




\begin{tabular}{|c|c|c|c|c|c|c|c|c|}
\hline \multirow{2}{*}{$\begin{array}{l}\text { Species } \\
\text { Lepidoptera }\end{array}$} & \multicolumn{4}{|c|}{$\begin{array}{c}\text { Beginning and ending of flight } \\
\text { (before midnight) } \\
\text { between }\end{array}$} & \multicolumn{4}{|c|}{$\begin{array}{c}\text { Ending of flight (before or } \\
\text { after midnight) } \\
\text { between }\end{array}$} \\
\hline & Lux & $T / N$ & Lux & $T / N$ & Lux & $T / N$ & Lux & $T / N$ \\
\hline Discestra trifolii Hfn. & 0.0279 & $\mathrm{~N}$ & 0.0025 & A & 0.0118 & $\mathrm{~N}$ & 0.0104 & $\mathrm{~N}$ \\
\hline Polia contigua Schiff. * & 0.0021 & A & 0.0021 & A & & & & \\
\hline Harmodia luteago Schiff. & 0.0484 & $\mathrm{~N}$ & 0.0039 & A & 0.0216 & $\mathrm{~N}$ & 0.0195 & $\mathrm{~N}$ \\
\hline Tholera decimalis Poda * & & & & & 0.0019 & $A$ & 0.0012 & $A$ \\
\hline Aplecta advena Schiff. & 0.0054 & $\mathrm{~A}$ & 0.0045 & A & 0.0019 & $A$ & 0.0071 & $A$ \\
\hline Xylomania conspicillaris L. & 0.1693 & $\mathrm{~N}$ & 0.0912 & $\mathrm{~N}$ & 0.0224 & $N$ & 0.0257 & $\mathrm{~N}$ \\
\hline Perigrapha i-cinctum Schiff. & 221.151 & $\mathrm{C}$ & 0.0211 & $\mathrm{~N}$ & 0.0089 & $\mathrm{~A}$ & 0.0088 & A \\
\hline Orthosia incerta Hfn. & 8507.98 & $\mathrm{D}$ & 676.8 & $\mathrm{D}$ & 0.0010 & $A$ & 0.0010 & $A$ \\
\hline Orthosia gothica L. & 206.548 & $\mathrm{C}$ & 0.0163 & $\mathrm{~N}$ & 0.0010 & $A$ & 0.0010 & $A$ \\
\hline Orthosia munda Schiff. & 206.548 & $\mathrm{C}$ & 0.0163 & $\mathrm{~N}$ & 0.0018 & A & 0.0019 & A \\
\hline Orthosia stabilis Schiff. & 2853.20 & $\mathrm{D}$ & 17.124 & $\mathrm{C}$ & 0.0207 & $\mathrm{~N}$ & 0.0209 & $\mathrm{~N}$ \\
\hline Orthosia miniosa F. * & 0.0005 & NS & 0.0005 & NS & & & & \\
\hline Orthosia cruda Schiff. & 206.548 & $\mathrm{C}$ & 0.0163 & $\mathrm{~N}$ & 0.0084 & $A$ & 0.0083 & $A$ \\
\hline Mythimna ferrago F. * & 0.0279 & $\mathrm{~N}$ & 0.0008 & NS & & & & \\
\hline Mythimna albipuncta Schiff. & 0.1450 & $\mathrm{~N}$ & 0.1003 & $\mathrm{~N}$ & 0.0072 & A & 0.0080 & A \\
\hline Mythimna 1-album Esp. & 0.0070 & $A$ & 0.0055 & $A$ & 0.0041 & $A$ & 0.0062 & $A$ \\
\hline Mythimna pallens L. & 0.3745 & $\mathrm{~N}$ & 0.0126 & $\mathrm{~N}$ & 0.0021 & A & 0.0021 & A \\
\hline Cucullia argentea Hfn. & 0.5654 & $\mathrm{~N}$ & 0.0014 & A & 0.3745 & $\mathrm{~N}$ & 0.0126 & $\mathrm{~N}$ \\
\hline Phlogophora meticulosa L. * & 0.0065 & A & 0.0065 & A & & & & \\
\hline Omphalophana antirrhini Hbn. * & 0.0604 & $\mathrm{~N}$ & 0.0216 & $\mathrm{~N}$ & & & & \\
\hline Calophasia lunula Hfn. $*$ & 0.0717 & $\mathrm{~N}$ & 0.0717 & $\mathrm{~N}$ & & & & \\
\hline Brachinochia sphinx Hfn. & 2.5005 & $\mathrm{~N}$ & 0.0024 & A & 0.0016 & $A$ & 0.0016 & $A$ \\
\hline Lithophane ornitopus Hfn. & 221.151 & $\mathrm{C}$ & 0.0211 & $\mathrm{~N}$ & 0.0010 & $A$ & 0.0010 & $A$ \\
\hline Meganephria oxyacanthe L. * & 0.0015 & A & 0.0015 & A & & & & \\
\hline Valeria oleagina Schiff. & 113.473 & $\mathrm{C}$ & 0.0139 & $\mathrm{~N}$ & 0.0017 & $A$ & 0.1383 & $N$ \\
\hline Crino satura Schiff. * & 16.6122 & $\mathrm{C}$ & 0.0134 & $\mathrm{~N}$ & & & & \\
\hline Agriopis convergens F. * & 0.0075 & A & 0.0046 & A & & & & \\
\hline Drybotodes protea Bkh. & 0.0075 & A & 0.0046 & A & 0.0302 & $N$ & 0.0900 & $N$ \\
\hline Antitype nigrocincta $\operatorname{Tr} . *$ & 0.1258 & $\mathrm{~N}$ & 0.0228 & A & & & & \\
\hline Eupsilia transversa Hfn. & 0.0228 & $\mathrm{~N}$ & 0.0234 & $\mathrm{~N}$ & 0.0014 & $A$ & 0.0012 & $A$ \\
\hline Eupsilia satellitia L. & 60.7056 & $\mathrm{C}$ & 0.0073 & A & 0.0014 & $A$ & 0.0014 & $A$ \\
\hline Conistra erythrocephala F. & 0.0234 & $\mathrm{~N}$ & 0.0292 & $\mathrm{~N}$ & 0.0005 & NS & 0.0005 & NS \\
\hline Conistra vau-punctatum Esp. & 0.0013 & A & 0.0014 & A & 0.0087 & $A$ & 0.0089 & $A$ \\
\hline Conistra vaccinii L. & 8507.98 & $\mathrm{D}$ & 676.80 & $\mathrm{D}$ & 0.0014 & A & 0.0014 & A \\
\hline Agrochola humilis Schiff. & 0.3661 & $\mathrm{~N}$ & 0.0083 & A & 0.0234 & $\mathrm{~N}$ & 0.0260 & $\mathrm{~N}$ \\
\hline Agrochola lychnidis Schiff. * & 0.0134 & $\mathrm{~N}$ & 0.0106 & $\mathrm{~N}$ & & & & \\
\hline Agrochola macilenta Hbn. & 0.0228 & $\mathrm{~N}$ & 0.0234 & $\mathrm{~N}$ & 0.0024 & A & 0.0014 & A \\
\hline Agrochola helvola L. * & 100.105 & $\mathrm{C}$ & 0.0369 & $\mathrm{~N}$ & & & & \\
\hline Agrochola litura L. & 0.0369 & $\mathrm{~N}$ & 0.0292 & $\mathrm{~N}$ & 0.0105 & $\mathrm{~N}$ & 0.0101 & $\mathrm{~N}$ \\
\hline
\end{tabular}




\begin{tabular}{|c|c|c|c|c|c|c|c|c|}
\hline \multirow{2}{*}{$\begin{array}{c}\text { Species } \\
\text { Lepidoptera }\end{array}$} & \multicolumn{4}{|c|}{$\begin{array}{c}\text { Beginning and ending of flight } \\
\text { (before midnight) } \\
\text { between }\end{array}$} & \multicolumn{4}{|c|}{$\begin{array}{c}\text { Ending of flight (before or } \\
\text { after midnight) } \\
\text { between }\end{array}$} \\
\hline & Lux & $T / N$ & Lux & $T / N$ & Lux & $T / N$ & Lux & $T / N$ \\
\hline Cosmia aurago F. * & 0.0165 & $\mathrm{~N}$ & 0.0017 & A & & & & \\
\hline Amphipyra pyramidea L. * & & & & & 0.0076 & $A$ & 0.0276 & $N$ \\
\hline Procus strigilis $\mathrm{Cl}$. & 0.0960 & $\mathrm{~N}$ & 0.0495 & $\mathrm{~N}$ & 0.0384 & $\mathrm{~N}$ & 0.0388 & $\mathrm{~N}$ \\
\hline Luperina testacea Schiff. & 0.0094 & A & 0.0083 & A & 0.0057 & A & 0.0045 & A \\
\hline Charanyca trigrammica Hfn. & 0.0976 & $\mathrm{~N}$ & 0.0178 & $\mathrm{~N}$ & 0.0029 & A & 0.0028 & A \\
\hline Cosmia trapezina L. & 0.0128 & $\mathrm{~N}$ & 0.0118 & $\mathrm{~N}$ & 0.0021 & A & 0.0021 & $\mathrm{~A}$ \\
\hline Apamea anceps Schiff. & 164.304 & $\mathrm{C}$ & 0.0738 & $\mathrm{~N}$ & 0.0203 & $N$ & 1.9993 & $N$ \\
\hline Dicycla oo L. & 0.0165 & $\mathrm{~N}$ & 0.0111 & $\mathrm{~N}$ & 0.0021 & $\mathrm{~A}$ & 0.0021 & A \\
\hline Heliothis maritima Grasl. * & 0.0008 & NS & 0.0009 & NS & & & & \\
\hline Chariclea delphinii L. * & 0.0484 & $\mathrm{~N}$ & 0.0039 & $\mathrm{~A}$ & & & & \\
\hline Lithacodia deceptoria Scop. * & & & & & 0.0229 & $N$ & 0.0468 & $N$ \\
\hline Erastria trabealis Scop. & 649.68 & $\mathrm{D}$ & 2.5273 & $\mathrm{~N}$ & 0.0495 & $\mathrm{~N}$ & 0.0453 & $\mathrm{~N}$ \\
\hline Tarache luctuosa Esp. & 0.0074 & A & 0.0057 & $\mathrm{~A}$ & 0.0018 & A & 0.0012 & $\mathrm{~A}$ \\
\hline Hylophila prasinana L. * & 0.0008 & NS & 0.0009 & NS & & & & \\
\hline Minucia lunaris Schiff. & 0.0738 & $\mathrm{~N}$ & 0.0065 & A & 0.0381 & $N$ & 0.1394 & $N$ \\
\hline Plusia chrysitis L. & 0.0105 & $\mathrm{~N}$ & 0.0101 & $\mathrm{~N}$ & 0.0006 & NS & 0.0003 & NS \\
\hline Abrostola trigemina Wern. * & & & & & 0.0207 & $N$ & 0.0229 & $N$ \\
\hline Autographa gamma L. & 0.0801 & $\mathrm{~N}$ & 0.0840 & $\mathrm{~N}$ & 0.0086 & $A$ & 0.0126 & $N$ \\
\hline Hadena confusa Hfn. * & 16.6122 & $\mathrm{C}$ & 0.0134 & $\mathrm{~N}$ & & & & \\
\hline Episema coeruleocephala L. & 18.0918 & $\mathrm{C}$ & 0.1921 & $\mathrm{~N}$ & 0.0320 & $N$ & 0.3020 & $N$ \\
\hline Toxocampa craccae F. * & 0.0057 & A & 0.0045 & A & & & & \\
\hline \multicolumn{9}{|l|}{ Lymantriidae } \\
\hline Dasychira fascelina L. * & & & & & 0.0010 & $A$ & 0.0026 & $A$ \\
\hline Dasyhira pudibunda L. & 0.0321 & $\mathrm{~N}$ & 0.0346 & $\mathrm{~N}$ & 0.0054 & $A$ & 0.0063 & $A$ \\
\hline Lymantria dispar L. & 15.1900 & $\mathrm{C}$ & 0.0042 & A & 0.0048 & $A$ & 0.3514 & $N$ \\
\hline \multicolumn{9}{|l|}{ Arctiidae } \\
\hline Gnophria rubricollis L. * & 0.0037 & $\mathrm{~A}$ & 0.0006 & NS & & & & \\
\hline Ocnogyna parasita Hbn. & 0.0017 & A & 0.0020 & A & 0.0005 & $N S$ & 0.0005 & $N S$ \\
\hline Phragmatobia fuliginosa $\mathrm{L}$. & 15.1900 & $\mathrm{C}$ & 0.0042 & $\mathrm{~A}$ & 0.0090 & A & 0.0074 & $\mathrm{~A}$ \\
\hline Spilosoma menthastri Esp. & 0.0363 & $\mathrm{~N}$ & 0.0165 & $\mathrm{~N}$ & 0.0034 & $\mathrm{~A}$ & 0.0008 & NS \\
\hline Eucharia costa Esp. & 0.0072 & A & 0.0080 & $\mathrm{~A}$ & 0.6542 & $N$ & 650.07 & $D$ \\
\hline Hyphantria cunea Drury * & 0.0034 & A & 0.0008 & NS & & & & \\
\hline Arctia villica L. & 0.0691 & $\mathrm{~N}$ & 0.0681 & $\mathrm{~N}$ & 0.0216 & $\mathrm{~N}$ & 0.0195 & $\mathrm{~N}$ \\
\hline \multicolumn{9}{|l|}{ Notodontidae } \\
\hline Stauropus fagi L. * & 0.0021 & A & 0.0023 & A & & & & \\
\hline Dicranura ulmi Schiff. & 0.5168 & $\mathrm{~N}$ & 0.0016 & $\mathrm{~A}$ & 0.0224 & $N$ & 0.0257 & $N$ \\
\hline Drymonia querna Schiff. $*$ & & & & & 0.0840 & $N$ & 0.0888 & $N$ \\
\hline Drymonia chaonia Hbn. & 11.9237 & $\mathrm{C}$ & 0.0037 & A & 0.0028 & A & 0.0006 & NS \\
\hline Pheosia tremula Clerck * & 0.0237 & $\mathrm{~N}$ & 0.0229 & $\mathrm{~N}$ & & & & \\
\hline
\end{tabular}




\begin{tabular}{|c|c|c|c|c|c|c|c|c|}
\hline \multirow{2}{*}{$\begin{array}{c}\text { Species } \\
\text { Lepidoptera }\end{array}$} & \multicolumn{4}{|c|}{$\begin{array}{c}\text { Beginning and ending of flight } \\
\text { (before midnight) } \\
\text { between }\end{array}$} & \multicolumn{4}{|c|}{$\begin{array}{c}\text { Ending of flight (before or } \\
\text { after midnight) } \\
\text { between }\end{array}$} \\
\hline & Lux & $T / N$ & Lux & $T / N$ & Lux & $T / N$ & Lux & $T / N$ \\
\hline $\begin{array}{l}\text { Notodonta phoebe Sieb. * } \\
\text { Ptilophora plumigera Esp. * } \\
\text { Phalera bucephala L. }\end{array}$ & $\begin{array}{l}0.0321 \\
0.0024 \\
0.0008 \\
\end{array}$ & $\begin{array}{c}\mathrm{N} \\
\mathrm{A} \\
N S\end{array}$ & $\begin{array}{l}0.0346 \\
0.0014 \\
0.0422 \\
\end{array}$ & $\begin{array}{l}\mathrm{N} \\
\mathrm{A} \\
N\end{array}$ & 0.8153 & $N$ & 611.76 & $D$ \\
\hline $\begin{array}{l}\text { Sphingidae } \\
\text { Marumba quercus Schiff. * } \\
\text { Mimas tiliae L. } \\
\text { Celerio euphorbiae L. * } \\
\text { Deilephila elpenor L. * } \\
\text { Pergesa porcellus L. }\end{array}$ & $\begin{array}{l}0.0976 \\
1.3611 \\
0.0018 \\
0.0003 \\
0.0476\end{array}$ & $\begin{array}{c}\mathrm{N} \\
\mathrm{C} \\
\mathrm{A} \\
\mathrm{NS} \\
\mathrm{N}\end{array}$ & $\begin{array}{l}0.0950 \\
0.0295 \\
0.0012 \\
0.0004 \\
0.0237\end{array}$ & $\begin{array}{c}\mathrm{N} \\
\mathrm{A} \\
\mathrm{NS} \\
\mathrm{N}\end{array}$ & 0.0012 & A & 0.0019 & A \\
\hline $\begin{array}{l}\text { Thyatiridae } \\
\text { Polyploca diluta F. } \\
\text { Polyploca flavicornis L. * } \\
\text { Polyploca ridens Hbn. }\end{array}$ & $\begin{array}{l}0.0075 \\
0.0067 \\
0.1693 \\
\end{array}$ & $\begin{array}{l}\mathrm{A} \\
\mathrm{A} \\
\mathrm{N}\end{array}$ & $\begin{array}{l}0.0046 \\
0.0006 \\
0.0912\end{array}$ & $\begin{array}{c}\text { A } \\
\mathrm{NS} \\
\mathrm{N}\end{array}$ & 0.0076 & $\begin{array}{l}A \\
\mathrm{~N}\end{array}$ & $\begin{array}{l}0.0276 \\
0.0381\end{array}$ & $\begin{array}{l}\mathrm{N} \\
N\end{array}$ \\
\hline $\begin{array}{l}\text { Drepanidae } \\
\text { Cylix glaucata Scop. * } \\
\text { Drepana binaria Hfn. } \\
\text { Asphalia ruficollis Schiff. }\end{array}$ & $\begin{array}{r}0.0009 \\
0.0295 \\
500.670\end{array}$ & $\begin{array}{l}\mathrm{NS} \\
\mathrm{N} \\
\mathrm{S}\end{array}$ & $\begin{array}{l}0.0009 \\
0.0321 \\
0.0652\end{array}$ & $\begin{array}{c}\mathrm{NS} \\
\mathrm{N} \\
\mathrm{N}\end{array}$ & $\begin{array}{l}0.0010 \\
0.0020\end{array}$ & $\begin{array}{l}\mathrm{A} \\
\mathrm{A}\end{array}$ & $\begin{array}{l}0.0010 \\
0.0140\end{array}$ & $\begin{array}{l}\mathrm{A} \\
\mathrm{N}\end{array}$ \\
\hline $\begin{array}{l}\text { Synthomidae } \\
\text { Amata phegea L. * } \\
\text { Dysauxes ancilla L. * }\end{array}$ & 0.0165 & $\mathrm{~N}$ & 0.0111 & $\mathrm{~N}$ & 0.0012 & $A$ & 0.0039 & $A$ \\
\hline Coleoptera & & & & & & & & \\
\hline $\begin{array}{l}\text { Melolonthidae } \\
\text { Melolontha melolontha L. * }\end{array}$ & $\begin{array}{l}676.8 \\
676.8\end{array}$ & $\begin{array}{l}\mathrm{D} \\
\mathrm{D}\end{array}$ & $\begin{array}{l}0.4073 \\
0.4073\end{array}$ & $\begin{array}{l}\mathrm{N} \\
\mathrm{N}\end{array}$ & & & & \\
\hline
\end{tabular}

\section{REFERENCES}

[1] Mazochin-Porshnyakov, G. A. (1956): The night into light of mercury vapour lamp and its application to entomology (in Russian). - Zool. Zhurn. 35: 238-244.

[2] Tshernyshev, V. B. (1961): Time of fly of the insects into light (in Russian). - Zool. Zhurn. 40(7): 1009-1018.

[3] Skuhravý, V., Zumr, V. (1981): Nocturnal and seasonal flight activity of the nun moth Lymantria monacha L. (Lepid. Lymantriidae) as determined by pheromone and light traps. - Z. ang. Ent. 92: 315-319.

[4] Dreisig, H. (1980): The importance of illumination level in the daily onset of flight activity in nocturnal moths. - Physiological Entomology. 5: 327-342.

[5] Nowinszky, L., Tóth, Gy. (1987): Influence of cosmic factors on the light-trap catches of harmful insects (in Hungarian). - Ph.D. Dissertation. Szombathely. 123. 